\title{
MODELAGEM CINÉTICA DE HIDROGENAÇÃO DE ESTIRENO UTILIZANDO CATALISADORES DE PALÁDIO
}

\author{
G. T. JUSTINO ${ }^{1}$, C. S. A. $\operatorname{VALE}^{1}$, M. A. P. da $\operatorname{SILVA}^{1}$ e A. R. SECCHI ${ }^{2}$ \\ ${ }^{1}$ Escola de Química/ UFRJ \\ ${ }^{2}$ Universidade Federal do Rio de Janeiro, COPPE, Programa de Engenharia Química \\ E-mail para contato: monica@eq.ufrj.br
}

\begin{abstract}
RESUMO - O alto nível de octanagem da gasolina de pirólise (PYGAS) leva à sua inserção no pool da gasolina. Contudo, sua utilização é dificultada pela presença de compostos formadores de goma, os quais são removidos através de hidrogenação. Avaliou-se a utilização de modelos cinéticos do tipo Langmuir-Hinshelwood para hidrogenação de estireno (uma molécula representativa da PYGAS), utilizando-se o catalisador $\mathrm{Pd} 10 \% \mathrm{Nb}_{2} \mathrm{O}_{5} / \mathrm{Al}_{2} \mathrm{O}_{3}$. Analisaram-se modelos que consideram a adsorção do hidrogênio dissociativa e não dissociativa, também observando a existência de um ou dois tipos de sítios catalíticos. Os experimentos foram realizados em reator batelada a temperatura e pressão constantes, variando-se as condições utilizadas na faixa de 16-56 bar, para temperatura de $80{ }^{\circ} \mathrm{C}$. Para avaliação dos modelos cinéticos, utilizaram-se os softwares MATLAB e EMSO. Observou-se que os modelos que propõem a adsorção de hidrogênio e compostos orgânicos no mesmo tipo de sítio resultaram em um melhor ajuste aos dados experimentais.
\end{abstract}

\section{INTRODUÇÃO}

A gasolina de pirólise (PYGAS) é um subproduto obtido a partir da pirólise da nafta de petróleo. É composta por hidrocarbonetos na faixa de C5-C12. Sua composição é majoritariamente de aromáticos do grupo chamado BTX (benzeno, tolueno e xilenos), sendo também considerável a presença de estireno e mono e di-olefinas. $\mathrm{O}$ alto nível de octanagem dessa mistura leva à sua inserção no pool da gasolina (Nijhuis et al., 2003).

Entretanto, a existência de compostos formadores de goma na PYGAS gera problemas à sua utilização no pool da gasolina. Além disso, recentes mudanças na legislação ambiental no país exigem menores níveis de hidrocarbonetos aromáticos na gasolina, tornando imprescindível a remoção desses compostos antes da utilização (Gaspar et al., 2008).

A melhor forma de remoção desses compostos indesejados é sua hidrogenação, a qual ocorre em duas etapas. No primeiro estágio, é realizada a hidrogenação seletiva de estireno e di-olefinas em condições moderadas. Já no segundo estágio, são removidos os compostos sulfurados e são hidrogenadas as mono-olefinas (Medeiros et al., 2007), utilizando condições mais severas de temperatura. No primeiro estágio, normalmente são utilizados catalisadores de paládio; enquanto no 


\section{9 a 22 de outubro de 2014 \\ Florianópolis/SC}

segundo, utilizam-se catalisadores como $\mathrm{CoMo} / \mathrm{Al}_{2} \mathrm{O}_{3}$, que são regeneráveis (Gaspar et al., 2008). Ainda na primeira etapa, nota-se que a hidrogenação de mono-olefinas é muito pouco significativa, visto que a seletividade à adsorção nos sítios catalíticos de paládio é muito maior para as di-olefinas em relação às mono-olefinas. Sendo assim, a hidrogenação das mono-olefinas só ocorre após a redução na concentração das di-olefinas.

Para representar a PYGAS, foi utilizada uma solução de estireno em tolueno. O estireno em particular foi selecionado como molécula-modelo da gasolina de pirólise por ser um de seus componentes menos reativos para hidrogenação (Nijhuis et al., 2003; Gaspar et al., 2008).

No presente trabalho, objetiva-se estudar a cinética de hidrogenação do estireno. Foram estimados os parâmetros cinéticos dos modelos de lei de potências e de Langmuir-Hinshelwood. Os experimentos foram feitos em reator batelada utilizando o catalisador $\mathrm{Pd} 10 \% \mathrm{Nb}_{2} \mathrm{O}_{5} / \mathrm{Al}_{2} \mathrm{O}_{3}$.

\section{PROCEDIMENTO EXPERIMENTAL}

\subsection{Preparo da Solução de Estireno}

O estireno foi previamente purificado para a eliminação do estabilizante terc-butil-catecol, que interfere na atividade do catalisador (Hoffer et al., 2004). O tolueno, empregado como solvente, foi purificado para a remoção de água, pois esta reduz a taxa de reação (Wolffenbuttel et al., 2001). Após os pré-tratamentos de estireno e de tolueno, foram preparadas soluções de estireno com concentração variando entre 0,3 e $0,8 \mathrm{~mol} / \mathrm{L}$. O preparo da solução de estireno era realizado antes de cada teste catalítico.

\subsection{Hidrogenação do Estireno}

As hidrogenações do estireno foram realizadas em um reator batelada (Parr Instruments) operando com agitação de $600 \mathrm{rpm}$ e temperatura e pressão de hidrogênio constantes. O catalisador $\mathrm{Pd} 10 \% \mathrm{Nb}_{2} \mathrm{O}_{5} / \mathrm{Al}_{2} \mathrm{O}_{3}$ (aproximadamente $0,0150 \mathrm{~g}$ ) foi previamente reduzido in situ sob pressão de hidrogênio puro $\left(10\right.$ bar) a $150{ }^{\circ} \mathrm{C}$ por $1 \mathrm{~h}$. Após a redução, o reator foi resfriado até temperatura ambiente. Então, foram adicionados $105 \mathrm{~mL}$ da mistura reacional sob atmosfera de $\mathrm{H}_{2}$ e o reator foi aquecido até a temperatura de reação. A seguir, foi retirada a alíquota zero, pressurizou-se o reator e se iniciou a agitação. Essas condições foram determinadas de forma a reduzir os efeitos difusivos. As alíquotas foram retiradas em intervalos de 5 min durante uma hora e analisadas em cromatógrafo HP $6890 \mathrm{~N}$ com coluna DB1 ( $\mathrm{L}=60 \mathrm{~m}, \mathrm{~d}_{\text {int }}=0,32 \mathrm{~mm}$ ) e dotado de detector de ionização de chama (FID). Os experimentos foram realizados a $80^{\circ} \mathrm{C}$ e a pressão de hidrogênio variou entre 16 e 56 bar.

\section{MODELAGEM CINÉTICA}

Foram avaliados dois modelos de lei de potências para a hidrogenação de estireno. As equações de taxa de reação são apresentadas na Tabela 1. 
O modelo 1A estima as ordens individuais para o estireno e o hidrogênio, já o modelo 1B considera que a ordem em relação ao estireno é um, estimando apenas a ordem da reação em relação à concentração de hidrogênio.

Tabela 1 - Modelos cinéticos de lei de potências avaliados

\begin{tabular}{|c|c|}
\hline Modelo & Equação da Taxa \\
\hline $1 \mathrm{~A}$ & $-r_{\text {est }}=k C_{\text {est }}{ }^{\alpha} C_{H_{\mathrm{n}}}{ }^{\beta}$ \\
\hline $1 \mathrm{~B}$ & $-r_{\text {est }}=k C_{e s t} C_{H_{\mathrm{n}}}{ }^{\beta}$ \\
\hline
\end{tabular}

Também foram avaliados modelos de Langmuir-Hinshelwood com três e quatro parâmetros, considerando a reação superficial dos compostos adsorvidos como etapa limitante do processo. As equações de taxa reacional para os modelos de Langmuir-Hinshelwood são apresentadas nas Tabelas 2 e 3, onde $k$ é a velocidade específica da reação e $K_{i}$ e $C_{i}$ são, respectivamente, a constante de adsorção e a concentração do composto $i$ (estireno, etilbenzeno ou hidrogênio) (Abreu et al., 2013).

Tabela 2 - Modelos cinéticos de Langmuir-Hinshelwood com três parâmetros

\begin{tabular}{|c|c|c|}
\hline Modelo & Equação da Taxa & $K_{\text {global }}$ \\
\hline $2 \mathrm{~A}$ & $-r_{e s t}=\frac{K_{g l o b a l} C_{H_{z}} C_{e s t}}{\left(1+K_{e s t} C_{e s t}+K_{e t b z} C_{e t b z}\right)^{2}}$ & $K_{\text {global }}=k K_{e s t} K_{H_{2}}$ \\
\hline $3 \mathrm{~A}$ & $-r_{e s t}=\frac{K_{g l o b a l} C_{H_{z}} C_{e s t}}{\left(1+K_{e s t} C_{e s t}+K_{e t b z} C_{e t b z}\right)^{3}}$ & $K_{\text {global }}=k K_{\text {est }} K_{H_{2}}$ \\
\hline $4 \mathrm{~A}$ & $-r_{e s t}=\frac{K_{g l o b a l} C_{H_{2}} C_{e s t}}{\left(1+K_{e s t} C_{e s t}+K_{e t b z} C_{e t b z}\right)}$ & $K_{\text {global }}=k K_{\text {est }} K_{H_{2}}$ \\
\hline $5 \mathrm{~A}$ & $-r_{e s t}=\frac{K_{g l o b a l} \sqrt{C_{H_{2}}} C_{e s t}}{\left(1+K_{e s t} C_{e s t}+K_{e t b z} C_{e t b z}\right)}$ & $K_{\text {global }}=k K_{\text {est }} \sqrt{K_{H_{z}}}$ \\
\hline $2 \mathrm{~B}$ & $-r_{e s t}=\frac{K_{g l o b a l} C_{H_{2}} C_{e s t}}{\left(1+K_{e s t} C_{e s t}+K_{H_{2}} C_{H_{2}}\right)^{2}}$ & $K_{\text {global }}=k K_{\text {est }} K_{H_{2}}$ \\
\hline $3 \mathrm{~B}$ & $-r_{e s t}=\frac{K_{g l o b a l} C_{H_{2}} C_{e s t}}{\left(1+K_{e s t} C_{e s t}+\sqrt{K_{H_{2}} C_{H_{2}}}\right)^{3}}$ & $K_{\text {global }}=k K_{e s t} K_{H_{z}}$ \\
\hline $4 \mathrm{~B}$ & $-r_{e s t}=\frac{K_{g l o b a l} C_{H_{2}} C_{e s t}}{\left(1+K_{e s t} C_{e s t}\right)\left(1+K_{H_{2}} C_{H_{2}}\right)}$ & $K_{\text {global }}=k K_{e s t} K_{H_{z}}$ \\
\hline $5 B$ & $-r_{\text {est }}=\frac{K_{\text {global }} \sqrt{C_{H_{2}}} C_{\text {est }}}{\left(1+K_{e s t} C_{e s t}\right)\left(1+\sqrt{K_{H_{2}} C_{H_{2}}}\right)}$ & $K_{\text {global }}=k K_{e s t} \sqrt{K_{H_{2}}}$ \\
\hline
\end{tabular}


Os modelos avaliados consideram a adsorção dos compostos orgânicos e do hidrogênio em um mesmo tipo de sítio catalítico (modelos 2 e 3) e em sítios distintos (modelos 4 e 5), assim como contemplam mecanismo de adsorção de hidrogênio dissociativo (modelos 3 e 5) e não-dissociativo (modelos 2 e 4).

Tabela 3 - Modelos cinéticos de Langmuir-Hinshelwood com quatro parâmetros

\begin{tabular}{|c|c|c|}
\hline Modelo & Equação da Taxa & $K_{\text {global }}$ \\
\hline $2 \mathrm{C}$ & $-r_{e s t}=\frac{K_{g l o b a l} C_{H_{z}} C_{e s t}}{\left(1+K_{e s t} C_{e s t}+K_{e t b z} C_{e t b z}+K_{H_{2}} C_{H_{2}}\right)^{2}}$ & $K_{\text {global }}=k K_{\text {est }} K_{H_{2}}$ \\
\hline $3 \mathrm{C}$ & $-r_{e s t}=\frac{K_{g l o b a l} C_{H_{2}} C_{e s t}}{\left(1+K_{e s t} C_{e s t}+K_{e t b z} C_{e t b z}+\sqrt{K_{H_{2}} C_{H_{2}}}\right)^{3}}$ & $K_{\text {global }}=k K_{e s t} K_{H_{2}}$ \\
\hline $4 \mathrm{C}$ & $-r_{e s t}=\frac{K_{\text {global }} C_{H_{2}} C_{\text {est }}}{\left(1+K_{\text {est }} C_{\text {est }}+K_{\text {etbz }} C_{\text {etbz }}\right)\left(1+K_{H_{2}} C_{H_{2}}\right)}$ & $K_{\text {global }}=k K_{e s t} K_{H_{2}}$ \\
\hline $5 \mathrm{C}$ & $-r_{e s t}=\frac{K_{\text {global }} \sqrt{C_{H_{2}}} C_{e s t}}{\left(1+K_{e s t} C_{e s t}+K_{e t b z} C_{e t b z}\right)\left(1+\sqrt{K_{H_{2}} C_{H_{2}}}\right)}$ & $K_{\text {global }}=k K_{e s t} \sqrt{K_{H_{2}}}$ \\
\hline
\end{tabular}

\section{RESULTADOS E DISCUSSÃO}

Para estimação dos parâmetros e a análise dos dados experimentais obtidos, foram utilizados os softwares MATLAB e EMSO (Soares e Secchi, 2003). A função objetivo utilizada foi a de mínimos quadrados. Os parâmetros foram estimados com base em 64 pontos experimentais, obtidos em 10 experimentos diferentes, e usando os métodos de busca aleatória adaptativa (Secchi e Perlingeiro, 1989) para busca global e dos poliedros flexíveis (Nelder e Mead, 1965) para refinamento da solução. A concentração de hidrogênio na fase líquida foi calculada a partir de um cálculo de flash simples, utilizando a equação de estado SRK (Zhou et al., 2006). O intervalo de confiança adotado foi de $95 \%$.

Para o modelo de lei de potências $1 \mathrm{~A}$, estimou-se a velocidade específica em $(8,8 \pm 8,3) \times 10^{2}$ $\mathrm{L}^{1,2} /\left(\mathrm{mol}^{0,2}\right.$ min $\left.\mathrm{g}_{\mathrm{cat}}\right)$. A ordem da reação foi estimada em $0,5 \pm 0,1$ para o estireno e $0,7 \pm 0,1$ para o hidrogênio, com função objetivo de 4875,02 e coeficiente de determinação igual a 0,880 . Já para o modelo $1 \mathrm{~B}$, estimou-se a velocidade específica em $(1,6 \pm 1,0) \times 10^{3} \mathrm{~L}^{1,7} /\left(\mathrm{mol}^{0,7} \mathrm{~min} \mathrm{~g}_{\text {cat }}\right)$ e a ordem da reação em relação ao hidrogênio em $0,7 \pm 0,1$. Esse último resultado foi igual ao do modelo $1 \mathrm{~A}$. A função objetivo para o modelo 1B assumiu o valor de 5933,04 e o coeficiente de determinação foi 0,855 . Observa-se que o erro referente à velocidade específica em ambos os modelos é muito elevado, além de apresentarem os mais baixos coeficientes de determinação de todos os modelos avaliados, sugerindo que modelo de lei de potências não descreve bem os dados experimentais. Os ajustes dos modelos de lei de potências aos dados podem ser observados na Figura 1.

As Tabelas 5 a 7 apresentam os parâmetros estimados para os modelos de LangmuirHinshelwood avaliados. Os modelos que consideraram a adsorção de compostos orgânicos e 
hidrogênio em um mesmo tipo de sítio catalítico proporcionaram um melhor ajuste aos dados.
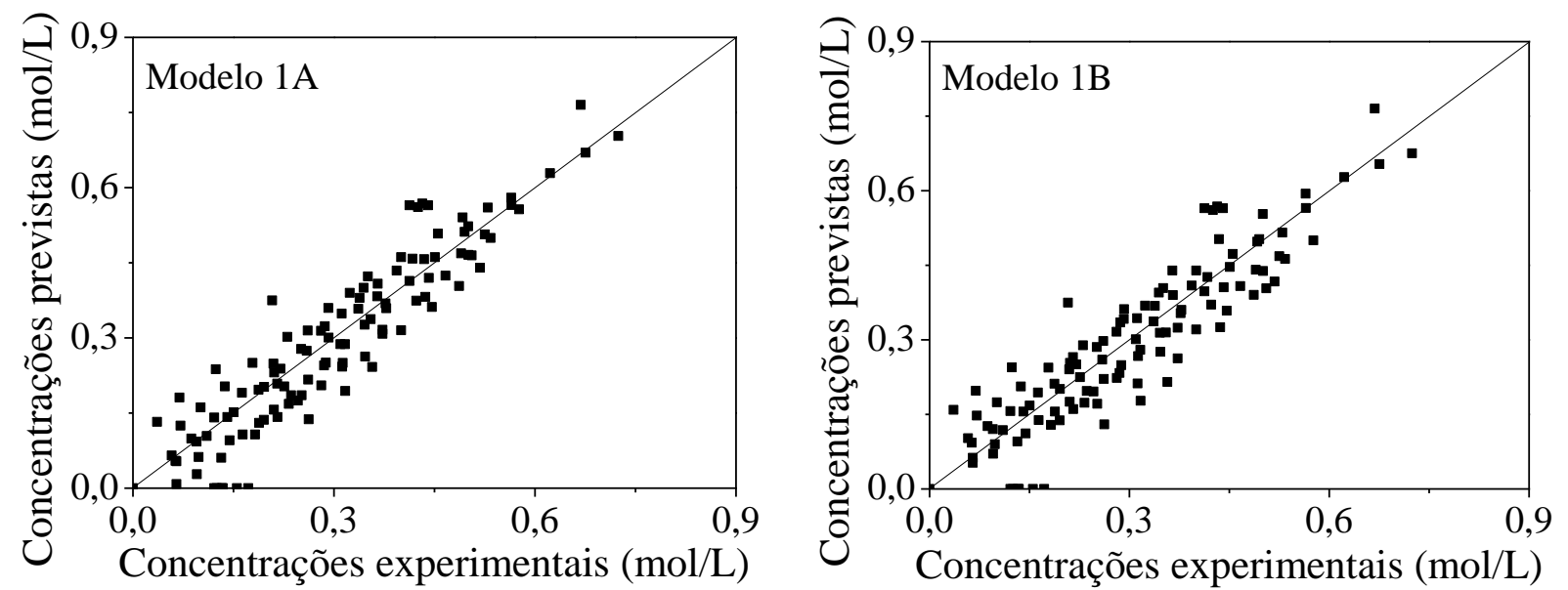

Figura 1 - Ajuste dos modelos de lei de potências aos dados experimentais.

Tabela 5 - Parâmetros estimados para os modelos de Langmuir-Hinshelwood 2A a 5A

\begin{tabular}{|c|c|c|c|c|c|}
\hline Modelo & $K_{\text {global }}{ }^{1}$ & $K_{\text {est }}(\mathrm{L} / \mathrm{mol})$ & $K_{e t b z}(\mathrm{~L} / \mathrm{mol})$ & $\begin{array}{l}\text { Função } \\
\text { Objetivo }\end{array}$ & $\mathrm{R}^{2}$ \\
\hline $2 \mathrm{~A}$ & $38,7 \pm 39,3$ & $4,4 \pm 3,1$ & $4,3 \pm 3,2$ & 3750,71 & 0,921 \\
\hline $3 \mathrm{~A}$ & $15,6 \pm 6,4$ & $\pm 0,4$ & $1,2 \pm 0,5$ & 3766,71 & 0,918 \\
\hline $4 \mathrm{~A}$ & $37,1 \pm 33,4$ & $20,3 \pm 19,1$ & $18,6 \pm 19,6$ & 3914,63 & 0,908 \\
\hline $5 \mathrm{~A}$ & $35,4 \pm 22,8$ & $73,0 \pm 47,8$ & $49,2 \pm 39,3$ & 4382,28 & 0,904 \\
\hline
\end{tabular}

${ }^{1}$ As unidades de $K_{\text {global }}$ estão em L ${ }^{2} /\left(\mathrm{mol} \mathrm{min} \mathrm{g}_{\text {cat }}\right)$ para os modelos $2 \mathrm{~A}$ a $4 \mathrm{~A}$ e $\mathrm{L}^{1,5} /\left(\mathrm{mol}^{0,5} \mathrm{~min} \mathrm{~g}_{\text {cat }}\right)$ para o modelo $5 \mathrm{~A}$.

Para os modelos 2A, 4A e 5A, observa-se que os erros associados a cada parâmetro são elevados, mostrando que esses modelos não apresentam significado estatístico. Já o modelo 3A apresenta um bom ajuste aos dados e parâmetros significativos estatisticamente, sendo o melhor dentre os modelos que consideram muito pequena a constante de equilíbrio de adsorção do hidrogênio.

Quanto aos modelos que consideram desprezível a constante de equilíbrio de adsorção do etilbenzeno, cujos resultados são apresentados na Tabela 6 , pode-se verificar que os modelos $2 \mathrm{~B}$ a $4 \mathrm{~B}$ apresentam significado estatístico. Contudo, quando comparados aos outros modelos de três parâmetros descritos na Tabela 5, verifica-se que não apresentam ajustes tão bons aos dados experimentais. Além disso, seus resultados para a constante de equilíbrio de adsorção do hidrogênio são maiores do que os valores encontrados para a constante de equilíbrio de adsorção do estireno, em desacordo com o encontrado na literatura (Zhou et al., 2007; 2010). Nesse aspecto, apenas o modelo 5B apresenta resultado de acordo com a literatura, mas a constante de equilíbrio de adsorção de hidrogênio não é um parâmetro significativo, dado o seu elevado intervalo de confiança. 
Quanto aos modelos de quatro parâmetros, verificou-se que os modelos $2 \mathrm{C}$ a $4 \mathrm{C}$ apresentam significado estatístico, enquanto o modelo $5 \mathrm{C}$ apresentou $K_{H_{2}}$ igual a zero e sem significado estatístico. Como se pode ver pelos valores da função objetivo e do coeficiente de determinação apresentados na Tabela 7, o modelo $2 \mathrm{C}$ é o que melhor representa os dados experimentais dentre os modelos de quatro parâmetros. Seus parâmetros também apresentaram alta significância estatística. Os ajustes dos modelos 2C a 4C são apresentados na Figura 2.

Tabela 6 - Parâmetros estimados para os modelos de Langmuir-Hinshelwood 2B a 5B

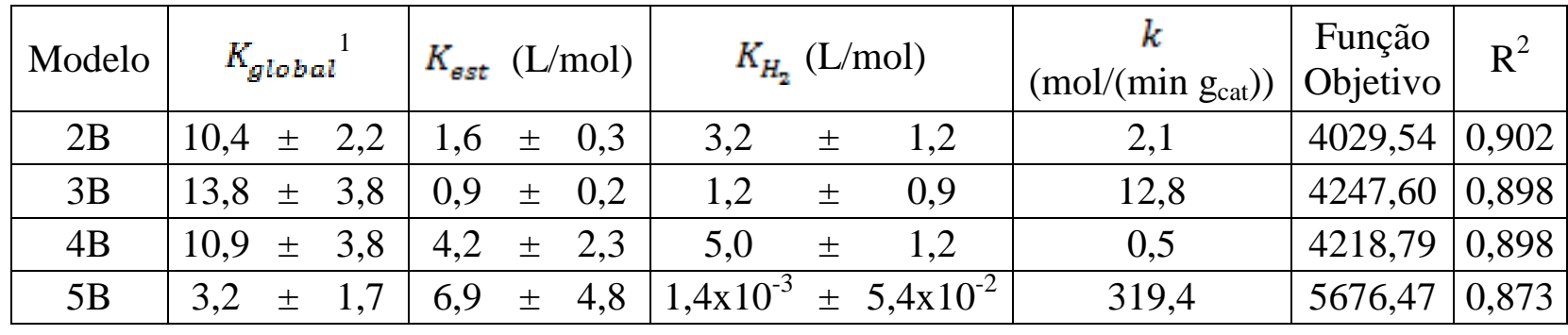

${ }^{1}$ As unidades de $K_{\text {global }}$ estão em L ${ }^{2} /\left(\mathrm{mol} \mathrm{min} \mathrm{g}_{\text {cat }}\right)$ para $2 \mathrm{~B}$ a $4 \mathrm{~B} \mathrm{e} \mathrm{L}{ }^{1,5} /\left(\mathrm{mol}^{0,5} \mathrm{~min}_{\mathrm{cat}}\right.$ ) para $5 \mathrm{~B}$.
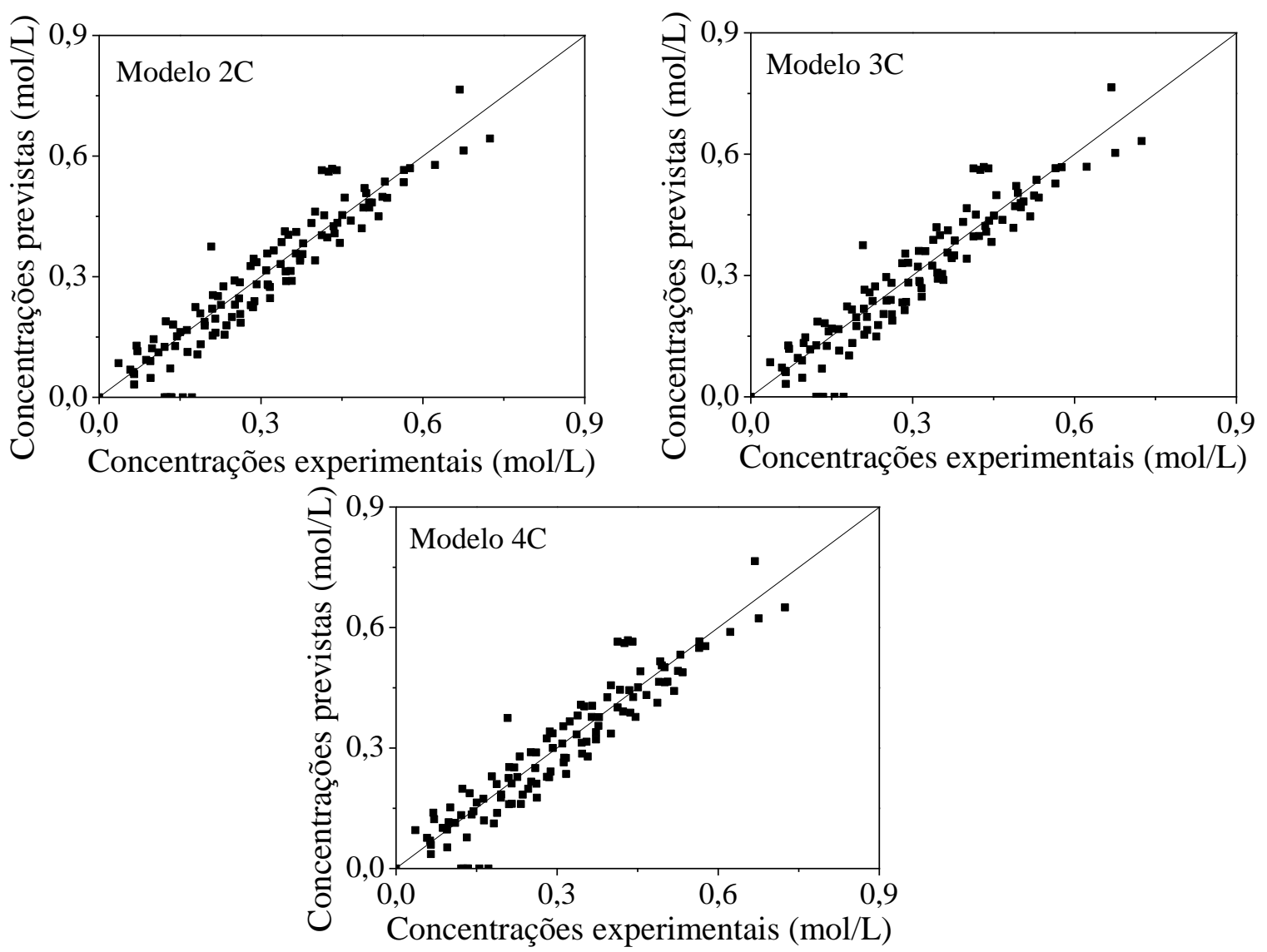

Figura 2 - Ajuste dos modelos de Langmuir-Hinshelwood de 4 parâmetros aos dados experimentais. 
Tabela 7 - Parâmetros estimados para os modelos de Langmuir-Hinshelwood de quatro parâmetros

\begin{tabular}{|c|c|cc|cc|c|c|c|c|}
\hline Modelo & $K_{\text {global }}$ & $K_{\text {est }}(\mathrm{L} / \mathrm{mol})$ & $K_{H_{2}}(\mathrm{~L} / \mathrm{mol})$ & $\begin{array}{c}K_{\text {etbz }} \\
(\mathrm{L} / \mathrm{mol})\end{array}$ & $\begin{array}{c}k \\
\left(\mathrm{~mol} /\left(\mathrm{min} \mathrm{g}_{\text {cat }}\right)\right.\end{array}$ & $\begin{array}{c}\text { Função } \\
\text { Objetivo }\end{array}$ & $\mathrm{R}^{2}$ \\
\hline 2C & $31,5 \pm 14,9$ & $3,6 \pm 1,4$ & 4,3 & $\pm 1,9$ & $2,2 \pm 0,9$ & 2,0 & 2985,77 & 0,934 \\
\hline 3C & $33,3 \pm 11,6$ & 1,6 & $\pm 0,5$ & 1,3 & $\pm 0,6$ & $1,2 \pm 0,5$ & 15,5 & 3256,19 & 0,929 \\
\hline 4C & $38,7 \pm 7,4$ & $16,7 \pm 6,0$ & 3,8 & $\pm 1,5$ & $10,7 \pm 2,7$ & 0,6 & 3162,72 & 0,927 \\
\hline 5C & $35,4 \pm 22,8$ & $73,0 \pm 47,8$ & 0 & $49,2 \pm 39,3$ & - & 4382,28 & 0,904 \\
\hline
\end{tabular}

${ }^{1}$ As unidades de $K_{\text {global }}$ estão em L ${ }^{2} /\left(\right.$ mol min $\left.\mathrm{g}_{\text {cat }}\right)$ para $2 \mathrm{C} \mathrm{a} 4 \mathrm{C} \mathrm{e} \mathrm{L}^{1,5} /\left(\mathrm{mol}^{0,5} \mathrm{~min}_{\mathrm{cat}}\right)$ para $5 \mathrm{C}$.

Observando os valores das constantes de equilíbrio de adsorção do estireno e do hidrogênio encontrados, pode-se compará-los com os valores calculados de acordo com os trabalhos de Zhou et al. $(2007 ; 2010)$, realizados na faixa de temperatura de $40-70{ }^{\circ} \mathrm{C}$ para catalisador de $\mathrm{Pd} / \mathrm{Al}_{2} \mathrm{O}_{3}$. Extrapolando-se seus resultados para a temperatura de $80{ }^{\circ} \mathrm{C}$, encontraram-se constantes de equilíbrio de adsorção iguais a $4 \mathrm{~L} / \mathrm{mol}$ e $32 \mathrm{~L} / \mathrm{mol}$ para o estireno. O primeiro resultado se assemelha ao encontrado para os modelos 2A, 2C e 4B, mas difere muito dos outros modelos analisados. Segundo os resultados obtidos por Nijhuis et al. (2003) com catalisador de $\mathrm{Pd} / \mathrm{Al}_{2} \mathrm{O}_{3}$, para a temperatura de $50{ }^{\circ} \mathrm{C}$ a constante de equilíbrio de adsorção do estireno é 13,3 $\pm 3,1 \mathrm{~L} / \mathrm{mol}$. Esse resultado é semelhante ao encontrado para o modelo 4C. Contudo, deve-se considerar que esses resultados foram obtidos a temperaturas mais baixas que as desse estudo e a constante de equilíbrio de adsorção se comporta de modo inversamente proporcional à temperatura.

Também segundo Zhou et al. (2007; 2010), a constante de equilíbrio de adsorção do hidrogênio assume valores de $1 \times 10^{-3} \mathrm{~L} / \mathrm{mol}$ e $4 \times 10^{-3} \mathrm{~L} / \mathrm{mol}$. Esses valores estão de acordo com o modelo $5 \mathrm{~B}$, mas são muito inferiores aos encontrados nesse trabalho para os outros modelos que consideram relevantes a adsorção do hidrogênio. Contudo, seus resultados podem ser comparados aos modelos que consideram baixo o valor desta constante, modelos $2 \mathrm{~A}$ a $5 \mathrm{~A}$.

As diferenças encontradas em relação aos trabalhos de Zhou et al. (2007; 2010) podem ser em decorrência das diferenças entre o catalisador utilizado nesse estudo ( $\mathrm{Pd} 10 \% \mathrm{Nb}_{2} \mathrm{O}_{5} / \mathrm{Al}_{2} \mathrm{O}_{3}$ em pó) e o utilizado por Zhou et al. $(2007 ; 2010)$, o qual era $\mathrm{Pd} / \mathrm{Al}_{2} \mathrm{O}_{3}$ do tipo egg-shell. Além disso, Zhou et al. (2007; 2010) estudaram a hidrogenação de uma mistura de compostos (dentre eles o estireno), na qual havia competição pelos sítios catalíticos, fator que pode modificar os parâmetros dos modelos.

\section{CONCLUSÕES}

Este trabalho avaliou modelos cinéticos do tipo lei de potências e Langmuir-Hinshelwood para a hidrogenação de estireno. Observou-se que os modelos de lei de potências não descrevem bem os dados experimentais. Dentre os modelos avaliados de Langmuir-Hinshelwood, os que apresentaram melhor ajuste aos dados experimentais foram os modelos $2 \mathrm{~A}$ e $2 \mathrm{C}$, os quais consideram a adsorção de compostos orgânicos e hidrogênio em um mesmo tipo de sítio catalítico e a adsorção de hidrogênio não-dissociativa. Entretanto, em ambos os casos, o valor obtido para a constante de equilíbrio de adsorção do hidrogênio se apresentou elevado quando comparado aos valores encontrados na 
literatura. Além disso, os modelos 3A e 3C, nos quais a adsorção do hidrogênio é dissociativa, apresentaram parâmetros estimados com melhores intervalos de confiança.

\section{AGRADECIMENTOS}

Ao CNPq, à ANP, à FINEP e ao PRH-13 pelo apoio financeiro.

\section{REFERÊNCIAS}

ABREU, B. M. N. B.; MODESTO, F. L. A; TRAVALlONI, L.; SILVA, M. A. P. Kinetic Modeling of Styrene Hydrogenation. EuropaCat-XI, 2013.

GASPAR, A. B.; SANTOS, G. R.; COSTA, R. S.; SILVA, M. A. P.; BRITTO, J. M. Hydrogenation of synthetic PYGAS-Effects of zirconia on $\mathrm{Pd} / \mathrm{Al}_{2} \mathrm{O}_{3}$. Catal. Today, v. 133, p. 140-145, 2008.

HOFFER, B.W.; BONNÉ, R. L. C.; LANGEVELD, A. D.; GRIFFITHS, C.; LOK, C. M.; MOULIJN, J. A. Enhancing the start-up of pyrolysis gasoline hydrogenation reactors by applying tailored ex situ presulfided $\mathrm{Ni} / \mathrm{Al}_{2} \mathrm{O}_{3}$ catalysts. Fuel, v. 83, p. 1-8, 2004.

MEDEIROS, J. L.; ARAÚJO, O. Q. F.; GASPAR, A. B.; SIlVA, M. A. P.; BRITTO, J. M. A. Kinetic model for the first stage of PYGAS upgrading. Braz. J. of Chem. Eng, v. 24, p. 119-133, 2007.

NELDER, J. A.; MEAD, R. A. Simplex Method for Function Minimization. The Comp. J., v. 7, n. 4, p. 308-313, 1965.

NIJHUIS, T. A.; DAUTZENBERG, F. M.; MOULIJN, J. A. Modeling of monolithic and tricklebed reactors for the hydrogenation of styrene. Chem. Eng. Sci., v. 58, p. 1113-1124, 2003.

SECCHI, A. R.; PERLINGEIRO, C. A. G. Otimização: Busca Aleatória Adaptativa. XII CNMAC, São José do Rio Preto, SP, p. 49-52. 1989.

SOARES, R. P.; SECCHI, A. R. EMSO: A New Environment for Modelling, Simulation and Optimization. ESCAPE 13, p. 947-952, 2003.

WOlfFEnBUTTEL, B. M. A.; NIJHUIS, T. A.; STANKIEWICZ, A.; MOULIJN, J. A. Influence of water on fast hydrogenation reactions with monolithic and slurry catalysts. Catal. Today, v. 69, p. 265-273, 2001.

ZHOU, Z.; CHENG, Z.; CAO, Y.; ZHANG, J.; YANG, D.; YUAN, W. Kinetics of the Selective Hydrogenation of Pyrolysis Gasoline. Chem. Eng. Technol., v. 30, p. 105-111, 2007.

ZHOU, Z.; CHENG, Z.; YANG, D.; ZHOU, X.; YUAN, W. Solubility of Hydrogen in Pyrolysis Gasoline. J. Chem. Eng., v. 51, p. 972-976, 2006.

ZHOU, Z.; ZENG, T.; CHENG, Z.; YUAN, W. Kinetics of selective hydrogenation of pyrolysis gasoline over an egg-shell catalyst. Chem. Eng. Sci., v. 65, p. 1832-1839, 2010. 